\title{
Platelet-Rich Plasma: Quantitative Assessment of Growth Factor Levels and Comparative Analysis of Activated and Inactivated Groups
}

\author{
Jeong Woo Lee ${ }^{1}$, O Hyun Kwon ${ }^{1}$, Taek Kyun Kim ${ }^{1}$, Young Kyoo Cho ${ }^{1}$, \\ Kang Young Choi ${ }^{1}$, Ho Yun Chung ${ }^{1}$, Byung Chae Cho ${ }^{1}$, Jung Dug Yang ${ }^{1}$, Jun Ho Shin ${ }^{2}$ \\ ${ }^{1}$ Department of Plastic and Reconstructive Surgery, Kyungpook National University School of Medicine, Daegu; ${ }^{2}$ Shin Jun Ho Plastic Surgery \\ Clinic, Seongnam, Korea
}

Background Platelet-rich plasma (PRP) has more concentrated platelets than normal plasma (approximately $150-400 \times 10^{3}$ cell/dL). Platelets excrete several growth factors and cytokines that are associated with the healing and regeneration process. However, even though PRP is widely used, the mechanism or actual effect is presently unclear. Therefore, this study was performed to investigate the levels of growth factors and platelet concentration rate.

Methods Autologous blood for preparing PRP was obtained from healthy subjects aged 25 to 35 years. The samples were divided into 4 experimental groups (inactivated whole blood, inactivated PRP, activated whole blood with thrombin and calcium chloride, and activated PRP). The platelet counts in the blood were analyzed and the growth factors were quantitatively measured. A statistical analysis was performed by using Dunn's multiple comparison test.

Results In the blood cell analysis, the platelet count of the PRP group was approximately 4.25 times higher than that of the whole blood group. In the quantitative analysis of growth factors, the platelet-derived growth factor (PDGF)-AB, PDGF-BB, and transforming growth factor- $\beta$ of the inactivated and activated PRP groups were higher than those of the inactivated and activated whole blood groups $(P<0.05)$.

Conclusions In this study, the platelet count and the levels of PDGF-AB and PDGF-BB in the PRP were determined. Further, more research is required on the bioactivity level of the growth factors secreted during the process of PRP preparation and the potency of growth factors that can be exerted physiologically in vivo.

Keywords Platelet-rich plasma / Platelet count / Platelet-derived growth factor / Transforming growth factors

Received: 11 Apr 2013 • Revised: 7 Jul 2013 • Accepted: 8 Jul 2013

pISSN: 2234-6163 • elSSN: 2234-6171 • http://dx.doi.org/10.5999/aps.2013.40.5.530 • Arch Plast Surg 2013;40:530-535
Correspondence: Jung Dug Yang Department of Plastic and Reconstructive Surgery, Kyungpook National University School of Medicine, 130 Dongdukro, Jung-gu, Daegu 700-721, Korea Tel: +82-53-420-5685 Fax: +82-53-425-3879 E-mail: lambyang@daum.net

No potential conflict of interest relevant to this article was reported.

\section{INTRODUCTION}

Platelet-rich plasma (PRP), which was first introduced in the field of oral and maxillofacial surgery by Whitman et al. [1] in 1997, has more concentrated platelets than normal plasma (approximately $150-400 \times 10^{3}$ cell $/ \mu \mathrm{L}$ ). Since it was reported that PRP accelerates the healing and regeneration of both soft and hard tissues, its scope of application has been continuously 
expanded to include various fields of surgery [2-4]. The concentrated platelets in PRP have been reported to play an important role in the wound healing process, aggregating rapidly at the damaged site and releasing a variety of growth factors and cytokines that are associated with wound healing, thereby accelerating the process of regenerating soft tissues and bone [3]. Examples of growth factors that are secreted from $\alpha$-particles by the activation of platelets include platelet-derived growth factor (PDGF), vascular-endothelial growth factor (VEGF), transforming growth factor- $\beta$ (TGF- $\beta$ ), insulin-like growth factor (IGF), and epidermal growth factor (EGF) $[5,6]$.

Based on these theoretical grounds and advantages, PRP is used as a source for autologous growth factors for various treatment purposes, such as implant placement and bone grafting, chronic wound cure, and fat transplantation $[1,4]$. However, even though PRP is widely used in clinics, the mechanism and actual effects are not completely understood. With numerous studies presenting contradictory results, the use of PRP remains controversial. Moreover, despite the importance of objectively evaluating the effectiveness of PRP through quantitative measurement of the levels of growth factors and platelets contained in the prepared PRP, there has been a lack of data and research related to the quantitative measurement of the constitutive components of PRP [4]. Thus, in order to determine the critical mechanism of PRP, and to enable an effective application in clinical settings, we sought to determine the necessity of activating PRP by using thrombin and calcium chloride for the quantitative measurement of its constitutive components, such as platelets and growth factors, and for its clinical application.

\section{METHODS}

\section{Experimental materials and methodology \\ Experimental materials \\ Blood collection}

For the experiment, $53 \mathrm{~mL}$ of whole blood (WB) was collected by piercing a blood vessel with a 21-gauge needle from a total of 20 healthy subjects consisting of 10 men and 10 women, aged 25 to 35 years. The collected samples were stored in containers with $7 \mathrm{~mL}$ of anti-coagulant citrate dextrose (Sigma, St. Louis, MO, USA).

\section{Preparation of PRP}

For centrifugation, the blood was injected into a $10 \mathrm{~mL}$ Thrombo kit (Korea Melsmon Co., Seongnam, Korea) and its mass was measured. Subsequently, a second Thrombo kit containing the same amount of a saline solution was prepared, and both kits were placed in a symmetrical position into the centrifuge (Fig. 1). After 3 minutes of centrifugation at 2,000 g, the Thrombo kit was removed and inserted in a push machine, the plasma layer and buffy coat were separated and collected, and the separated plasma layer was reinserted into the Thrombo kit. After calibrating the weight symmetry, the sample was again centrifuged for 5 minutes at 5,000 g, it was inserted into the push machine, and

\section{Fig 1. Gross photo of the PRP manufacturing system}

The platelet-rich plasma (PRP) manufacturing system is composed of three kits and facilitates accurate extraction of the PRP. (A) Thrombo kit, (B) Centrifuge, $(C)$ Extraction of PRP.
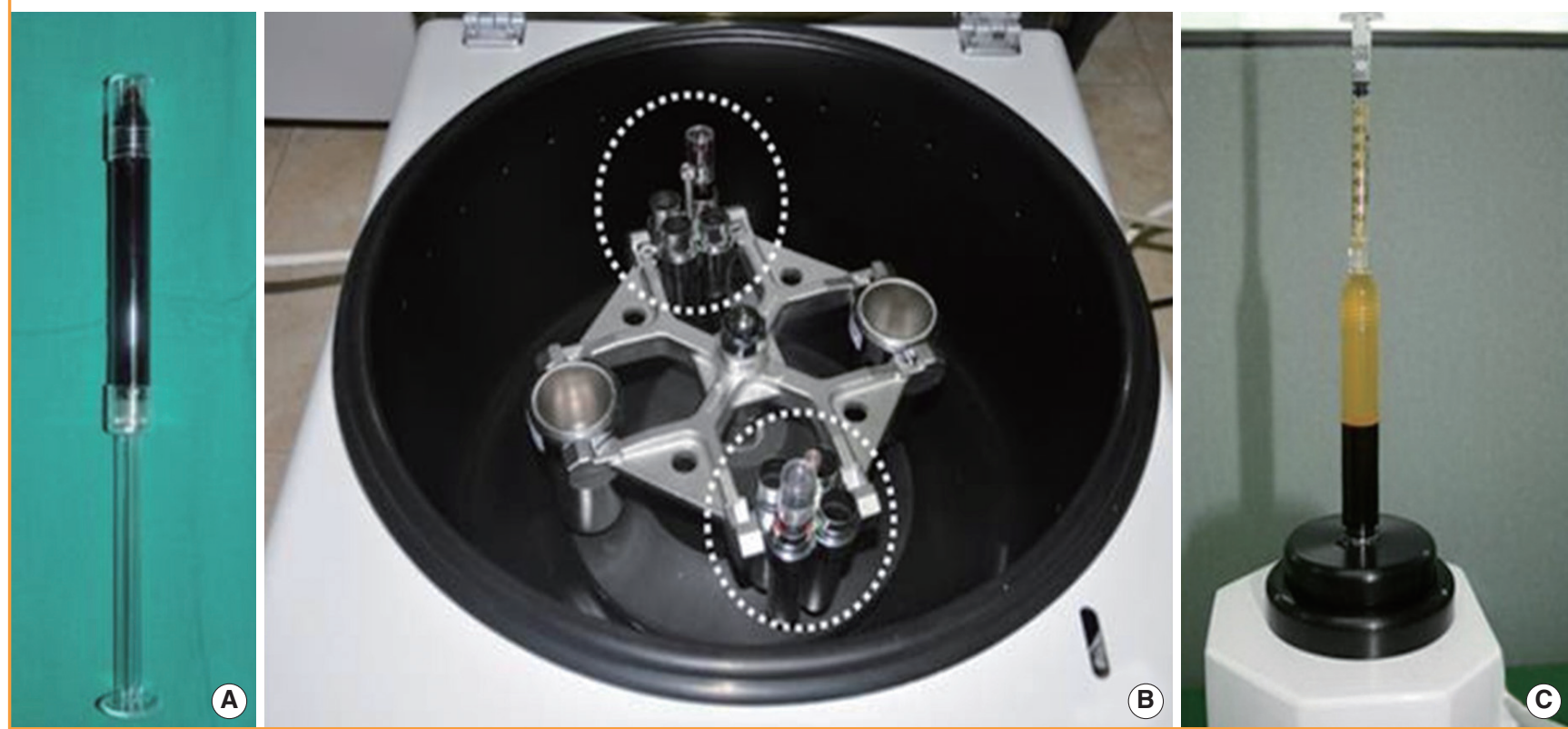
the platelet-rich plasma was harvested from the lowest layer (Fig. 1).

\section{Experimental methodology}

To compare the PRP and WB and analyze the constitutive components according to activation, the following 4 experimental groups were made: inactivated $\mathrm{WB}$, inactivated PRP, WB activated with thrombin and calcium chloride, and PRP activated with thrombin and calcium chloride.

To perform the activation, we mixed thrombin powder (Dirabine, Korea United Pharmaceutical Inc., Seoul, Korea) and calcium chloride (Kalmia, Korea United Pharmaceutical) with the prepared whole blood and PRP to reach $137.93 \mathrm{U} / \mathrm{mL}$ and $13.793 \mathrm{mg} / \mathrm{mL}$, respectively.

Group A, inactivated WB experimental group (WB with no treatment); group $\mathrm{B}$, activated $\mathrm{WB}$ experimental group $(\mathrm{WB}+$ thrombin $+\mathrm{CaCl})$; group $\mathrm{C}$, inactivated PRP experimental group (PRP with no treatment); group $\mathrm{D}$, activated PRP experimental group $(\mathrm{PRP}+$ thrombin $+\mathrm{CaCl})$.

\section{Experimental assessment}

General blood test

General blood testing was performed using an automated blood tester, Siemens Advia 2120i (Siemens Healthcare Diagnostics Inc., Erlangen, Germany) on the specimens obtained from the WB and PRP experimental groups prepared by centrifugation after mixing $0.1 \mathrm{~mL}$ of it with $0.9 \mathrm{~mL}$ of a saline solution. The number of platelets, red blood cells, neutrophils, lymph cells, and monocytes in the WB and PRP were measured, and the concentration factor (\%) of the baseline blood was calculated.

\section{Quantitative measurement of growth factors using enzyme-} linked immunosorbent assay (ELISA)

The levels of platelet-derived growth factors (PDGF), PDGF$\mathrm{AB}, \mathrm{PDGF}-\mathrm{BB}$, and transforming growth factor-beta (TGF- $\beta$ ) were measured using ELISA analysis for the quantitative measurement of growth factors contained in the PRP.

\section{PDGF-AB (hetero-dimer)}

For a quantitative analysis of growth factors, the concentrations of the growth factors released from the inactivated $\mathrm{WB}$ and PRP and from the WB and PRP activated with thrombin and calcium chloride were analyzed. In order to analyze the levels of PDGF$\mathrm{AB}$, the Quantikine Enzyme-linked Immunosorbent Assay Kit (R\&D Systems Inc., Minneapolis, MN, USA) was used. The quantitative analysis of the growth factors was conducted according to the instructions of the manufacturer. A capture antibody specific to the protein was diluted to $1.0 \mu \mathrm{g} / \mathrm{mL}$ in PBS, smeared onto 96-well microplates (100 $\mu \mathrm{L}$ each), and cultivated for 1 day. The surface was washed down to remove any unbound antibodies, and a non-specific reaction was suppressed using $400 \mu \mathrm{L}$ of reagent diluent. A total of $100 \mu \mathrm{L}$ of each standard solution and sample was injected in each well, cultivated for 2 hours, washed thoroughly, and subsequently diluted. Detection antibodies of PDGF-AB were injected again, left to react for 2 hours, and washed. After the reaction was induced by adding streptavidin-horseradish peroxidase (HRP), it was washed down; the bonded peroxidase of the same amount with the remaining protein was retained. A reaction was induced for about 20 minutes using a substrate solution (tetramethylbenzene and hydrogen peroxide), and a stop solution (sulfuric acid) was added to terminate the reaction. The optical density of each well was measured at a 450-nm wavelength using a microplate reader.

\section{PDGF-BB (homo-dimer)}

The quantitative analysis was conducted using the same ELISA kit used for the hetero-dimer PDGF-AB, and the antibody that specifically reacts with $\mathrm{PDGF}-\mathrm{BB}$ protein was used. The test was performed after diluting the concentration in accordance with the protein characteristics of PDGF-BB.

\section{TGF- $\beta$}

A quantitative analysis was conducted using the same ELISA kit used for the PDGF, and the antibody that specifically reacts with TGF- $\beta$ protein was used. The test was performed by diluting the concentration in accordance with the characteristics of TGF- $\beta$.

\section{Statistical analysis}

The measured values of the growth factors of the experimental groups were compared using Dunn's multiple comparison test $(\mathrm{P}<0.05)$.

\section{RESULTS}

\section{Blood analysis test}

The results of the blood analysis of the WB and PRP revealed that the number of red blood cells, white blood cells, neutrophils, lymph cells, and monocytes did not show any statistical differences (Table 1). The mean number of platelets was $201.16 \times 10^{3}$ cell $/ \mu \mathrm{L}$ for the inactivated WB experimental group, $894.74 \times 10^{3}$ cell $/ \mu \mathrm{L}$ for the inactivated PRP experimental group, which was 4.25 times higher than that of $\mathrm{WB}$; it was $199.83 \times 10^{3}$ cell $/ \mu \mathrm{L}$ for the activated WB experimental group and $901.61 \times 10^{3}$ cell/ $\mu \mathrm{L}$ for the activated PRP experimental group, which was about 4.51 times higher than that of the WB $(\mathrm{P}<0.05)$ (Table 1$)$. 
Table 1. Comparison of the whole blood and PRP

\begin{tabular}{|lcc|}
\hline Characteristic & $\begin{array}{c}\text { Whole blood } \\
\left(\text { cell } \times 10^{3} / \mu \mathrm{L}\right)\end{array}$ & $\begin{array}{c}\text { PRP } \\
\left(\text { cell } \times 10^{3} / \mu \mathrm{L}\right)\end{array}$ \\
\hline Platelet & $201.16 \pm 58.33^{\text {a) }}$ & $894.74 \pm 205.79^{\mathrm{a})}$ \\
WBC & $5.163 \pm 1.96$ & $4.467 \pm 1.83$ \\
Neutrophil & $3.072 \pm 1.13$ & $0.813 \pm 0.32$ \\
Lymphocyte & $1.926 \pm 0.59$ & $2.217 \pm 0.84$ \\
Monocyte & $0.394 \pm 0.13$ & $0.401 \pm 0.15$ \\
RBC & $0.328 \pm 0.12$ & $0.175 \pm 0.07$ \\
\hline
\end{tabular}

PRP, platelet-rich plasma; WBC, white blood cell; RBC, red blood cell.

The numbers of WBCs, neutrophils, lymphocytes, monocytes, and RBCs did not differ statistically between whole blood and PRP. However, the number of platelets significantly increased in the PRP group ( $\left.{ }^{(a)} P<0.05\right)$.

\section{Quantitative analysis of growth factors PDGF-AB}

PDGF-AB level in the inactivated WB experimental group was found to be $5.69 \pm 0.94 \mathrm{ng} / \mathrm{mL}$, and that in the inactivated PRP experimental group was found to be $47.13 \pm 0.94 \mathrm{ng} / \mathrm{mL}$, which was 8.28 times higher than that in the $\mathrm{WB}$, and this was statistically significant $(\mathrm{P}<0.05)$ (Fig. 2$)$. In addition, the PDGF-AB level in the activated $\mathrm{WB}$ experimental group was found to be $9.78 \pm 0.97 \mathrm{ng} / \mathrm{mL}$, and the PDGF-AB level in the activated PRP experimental group was found to be $50.21 \pm 2.75 \mathrm{ng} / \mathrm{mL}$, which was 5.13 times higher than that of the inactivated PRP, and was also statistically significant (Fig. 2$)(\mathrm{P}<0.05)$. The level in the activated PRP group was 1.06 times higher than that in the inactivated PRP group, but no statistically significant difference was found (Fig. 3).

\section{PDGF-BB}

The PDGF-BB level in the inactivated WB experimental group was found to be $3.97 \pm 0.43 \mathrm{ng} / \mathrm{mL}$, and the PDGF-BB level in the inactivated PRP experimental group was found to be $36.23 \pm 1.01 \mathrm{ng} / \mathrm{mL}$, which was 9.12 times higher than that in the $\mathrm{WB}$, showing a statistically significant difference $(\mathrm{P}<0.05)$ (Fig. 2). In addition, the PDGF-BB level in the activated WB experimental group was found to be $6.12 \pm 0.73 \mathrm{ng} / \mathrm{mL}$, and the PDGF-BB level in the activated PRP experimental group was found to be $37.15 \pm 1.62 \mathrm{ng} / \mathrm{mL}$, which was 6.07 times higher than that in the PRP, showing statistical significance $(\mathrm{P}<0.05)$ (Fig. 2). The number in the PRP group activated with thrombin and calcium chloride was 1.02 times higher than that in the inactivated PRP group; however, this difference was not statistically significant (Fig. 3).

\section{TGF- $\beta$}

The TGF- $\beta$ level in the inactivated WB experimental group was found to be $47.09 \pm 1.35 \mathrm{ng} / \mathrm{mL}$, and the TGF- $\beta$ level in the inactivated PRP experimental group was found to be $120.28 \pm 1.74$
Fig 2. The comparison between whole blood and inactivated PRP

The amounts of platelet-derived growth factor (PDGF)-AB, PDGF-BB, and transforming growth factor (TGF)- $\beta$ increased significantly in the platelet-rich plasma (PRP) group (Dunn's multiple comparison test, $\left.{ }^{a)} \mathrm{P}<0.05\right)$.

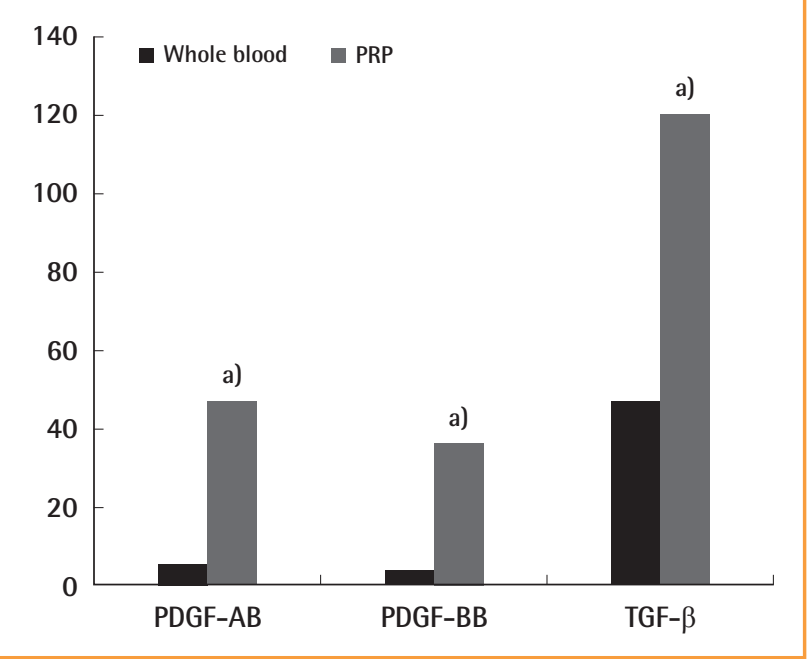

Fig 3. A comparison of inactivated and activated PRP

The difference between the inactivated PRP and activated PRP groups did not reach statistical significance (Dunn's multiple comparison test, $P>0.05)$. PRP, platelet-rich plasma; PDGF, platelet-derived growth factor; TGF- $\beta$, transforming growth factor.

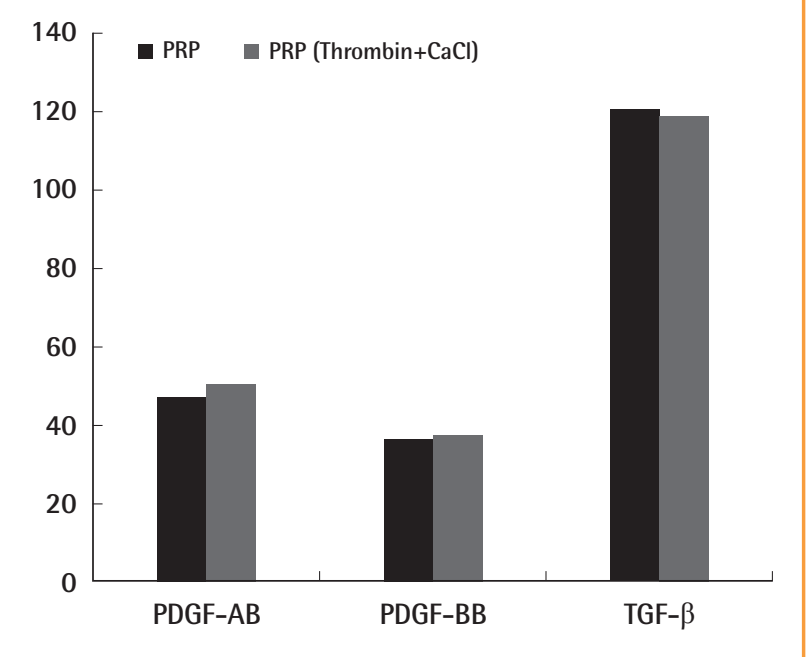

$\mathrm{ng} / \mathrm{mL}$, which was 2.55 times higher than that in the WB. The TGF- $\beta$ level in the activated WB experimental group was found to be $45.08 \pm 0.92 \mathrm{ng} / \mathrm{mL}$, and the TGF- $\beta$ level in the activated PRP experimental group was found to be $118.7 \pm 1.84 \mathrm{ng} / \mathrm{mL}$, which was 2.63 times higher than that in the PRP. Statistical significance was confirmed for each case $(\mathrm{P}<0.05)$ (Fig. 2). The values for the PRP group activated with thrombin and calcium chloride were lower than those for the inactivated PRP group (Fig. 3). 


\section{DISCUSSION}

PRP is autologous plasma containing highly concentrated platelets, and plasma containing a large amount of platelets possesses abundant growth factors, enabling healing and regeneration of tissues. The tissues of the human body, when damaged, are regenerated and healed by cell growth and its redistribution and rebuilding, and the following 3 factors play important roles therein. The first factor is a scaffold that forms a framework; the second factor is undifferentiated cells; and the third is a growth factor that is a signal protein secreted from the platelets, plasma, and white blood cells. Among the 3 factors, growth factors play the most important role. It is reported that growth factors are contained in the highest concentrations in the platelets, and that they play a pivotal role during the wound healing process [7-12]. For the growth factors contained in the platelets, when damages such as wounds or inflammation occur in tissues, the platelets are activated and the growth factors are secreted from the inner $\alpha$-particles, including the 3 types of PDGF (PDGFAA, PDGF-AB, and PDGF-BB) and 2 types of TGF- $\beta$ as well as IGF and EGF $[5,13,14]$. In particular, of those growth factors, PDGF and TGF- $\beta$ play critical roles in the regeneration and healing of the damaged tissues.

PDGF is released from the platelets and plays important roles in relation to various cells. In our study, the level of growth factors in each experimental group was analyzed and compared, and it was found that the PDGF-AB level was 8.28 times higher in the inactivated PRP experimental group than that in the $\mathrm{WB}$, and it was 5.13 times higher in the activated PRP experimental group than in the WB. Further, the PDGF-BB level was 9.12 times higher in the inactivated PRP experimental group than in the $\mathrm{WB}$, and it was 6.07 times higher in the activated PRP experimental group than in the WB. However, Weibrich et al. [15] reported that PDGF growth factors were at least 5 times higher in the activated PRP than in the inactivated PRP. However, in our study, the PDGF-AB level was increased by only 1.06 times compared with that in the inactivated PRP, showing no statistically significant difference $(\mathrm{P}>0.05)$. Further, the comparative analysis of the PDGF-BB value between the activated and inactivated $P R P$ groups did not show statistical significance $(P>0.05)$.

TGF- $\beta$, another growth factor in PRP, is a multi-functional potential cytokine involved in the control of cell growth, stimulation of matrix production, and suppression of the immune system. In our study, the analysis and comparison of the level of growth factors in each experimental group revealed that the TGF- $\beta$ value was 2.55 times higher in the inactivated PRP experimental group than in the inactivated $\mathrm{WB}$, and it was 2.63 times higher in the activated PRP experimental group than in the activated WB, showing statistical significance. Further, the mean TGF- $\beta$ level in the inactivated PRP group was found to be $120.28 \pm 1.74 \mathrm{ng} / \mathrm{mL}$, and that in the activated PRP group was $118.7 \pm 1.84 \mathrm{ng} / \mathrm{mL}$, revealing that the concentration of TGF- $\beta$ was rather decreased in the activated experimental group.

As shown above, our study and that of Roussy et al. [16] gave different results, and those of other prior analysis regarding the quantitative measurement of the growth factors contained in PRP also varied [15]. Such differences in the quantitative analysis of growth factors seems to be multifactorial, and include inter-patient variability of the amount of proteins contained in the platelets, different degrees of concentration of platelets during PRP preparation, activation, or inactivation, as well as different degrees of platelet membrane breakage and the degree of platelet activation at the time of measurement [12].

In 2004, Marx et al. [9] suggested that $1 \times 10^{6}$ platelet $/ \mu \mathrm{L}$ be set as the threshold concentration of therapeutic PRP in order to ensure a therapeutically effective amount of growth factors in PRP, which are important for tissue regeneration and healing. In our study, the concentration factor of platelets was found to be about 4.25 times higher in the inactivated PRP experimental group than in the inactivated WB; in the comparative experiments between the activated $\mathrm{WB}$ and $\mathrm{PRP}$, this value was 4.51 times higher in the PRP experimental group than in the WB experimental group. Accordingly, the efficiency of platelet collection in our study was confirmed to be consistent with the effective PRP suggested by Marx et al. [9], and therein, it was confirmed that activation with thrombin and calcium chloride does not have any relationship with the efficiency of platelet collection. Moreover, as a result of comparative analysis of the efficiency of platelet collection and the level of PDGF-AB, PDGF-BB, and TGF- $\beta$ before and after the activation of PRP in our study, it was confirmed that there was no statistical difference. This indicates that there is no influence of activation of PRP using thrombin and calcium chloride on the secretion of growth factors and on the amount of platelets concentrated in plasma. As such, it was inferred that adding thrombin and calcium chloride is not necessary for the effective preparation of PRP or the release of growth factors. Concerning the causes for the activation of platelets other than that by thrombin and calcium chloride, the centrifugal force applied for the separation of PRP is worth considering. Marx et al. [9] suggested a 2 -step separation process, and reported that the total rotation should be about $11,000 \mathrm{~g}$ including separating rotations and concentrating rotations, which corresponds to about one third of the force required for breaking the membrane of the platelets $(30,000 \mathrm{~g})[17]$. Taking this as a reference, the recommended rate of centrifugation in our PRP manufacturing system is a to- 
tal rotation of $31,000 \mathrm{~g}$ consisting of the separating rotations of $2,000 \mathrm{~g}$ for 3 minutes and the concentrating rotations of 5,000 $\mathrm{g}$ for 5 minutes, which is similar to the force capable of breaking the platelet membranes as suggested by Marx et al. [9]. Accordingly, it was inferred that the platelet membranes were damaged by the high-speed centrifugation in the absence of PRP activation using thrombin and calcium chloride, influencing the activation of platelets, and as such, the growth factors are released. Second, the process of cultivation for 1 hour at $37^{\circ} \mathrm{C}$ can also be taken into consideration. It was inferred that as PRP contains fibrinogen, the amount of growth factors, like in the case where calcium and thrombin were added, can be released by cultivation for an appropriate period of time.

Through the present experiments, we quantified the platelets and growth factors in PRP and confirmed a change in the growth factors during the manufacturing process. In conclusion, the concentration of platelets was statistically significantly higher in $\mathrm{PRP}$ than in $\mathrm{WB}$, and the levels of growth factors, i.e., PDGF$A B, P D G F-B B$, and TGF- $\beta$, were also significantly higher in the PRP than in the WB. Moreover, through the analysis of the change of growth factors according to the activation using thrombin and calcium chloride, it was confirmed that no statistical relationships exist among any of the WB and PRP experimental groups. Such experimental results suggest both objective and theoretical grounds in effectively applying PRP in clinical settings, and it is believed that further studies are required to establish a standard manufacturing method that can stabilize the release of growth factors and produce an appropriate amount of growth factors to effectively stimulate the regeneration and healing of tissues in vivo.

\section{REFERENCES}

1. Whitman DH, Berry RL, Green DM. Platelet gel: an autologous alternative to fibrin glue with applications in oral and maxillofacial surgery.J Oral Maxillofac Surg 1997;55:1294-9.

2. Bhanot S, Alex JC. Current applications of platelet gels in facial plastic surgery. Facial Plast Surg 2002;18:27-33.

3. Margolis DJ, Kantor J, Santanna J, et al. Effectiveness of platelet releasate for the treatment of diabetic neuropathic foot ulcers. Diabetes Care 2001;24:483-8.

4. Marx RE, Carlson ER, Eichstaedt RM, et al. Platelet-rich plasma: Growth factor enhancement for bone grafts. Oral
Surg Oral Med Oral Pathol Oral Radiol Endod 1998;85: 638-46.

5. Kevy SV, Jacobson MS. Comparison of methods for point of care preparation of autologous platelet gel.J Extra Corpor Technol 2004;36:28-35.

6. Slater M, Patava J, Kingham K, et al. Involvement of platelets in stimulating osteogenic activity. J Orthop Res 1995;13: 655-63.

7. Kim ES, Park EJ, Choung PH. Platelet concentration and its effect on bone formation in calvarial defects: an experimental study in rabbits. J Prosthet Dent 2001;86:428-33.

8. Weibrich G, Kleis WK, Hafner G. Growth factor levels in the platelet-rich plasma produced by 2 different methods: curasan-type PRP kit versus PCCS PRP system. Int J Oral Maxillofac Implants 2002;17:184-90.

9. Marx RE. Platelet-rich plasma: evidence to support its use. J Oral Maxillofac Surg 2004;62:489-96.

10. Min SK, Kim HJ, Cha SR. Study on platelet rich plasma concentration according to processing method. J Korean Assoc Maxillofac Plast Reconstr Surg 2005;27:24-31.

11. Everts PA, Brown Mahoney C, Hoffmann JJ, et al. Plateletrich plasma preparation using three devices: implications for platelet activation and platelet growth factor release. Growth Factors 2006;24:165-71.

12. Pietrzak WS, Eppley BL. Platelet rich plasma: biology and new technology.J Craniofac Surg 2005; 16:1043-54.

13. Hosgood G. Wound healing. The role of platelet-derived growth factor and transforming growth factor beta. Vet Surg 1993;22:490-5.

14. Spencer EM, Tokunaga A, Hunt TK. Insulin-like growth factor binding protein-3 is present in the alpha-granules of platelets. Endocrinology 1993;132:996-1001.

15. Weibrich G, Kleis WK, Buch R, et al. The Harvest Smart PRePTM system versus the Friadent-Schutze platelet-rich plasma kit. Clin Oral Implants Res 2003;14:233-9.

16. Roussy Y, Bertrand Duchesne MP, Gagnon G. Activation of human platelet-rich plasmas: effect on growth factors release, cell division and in vivo bone formation. Clin Oral Implants Res 2007;18:639-48.

17. Leitner GC, Gruber R, Neumuller J, et al. Platelet content and growth factor release in platelet-rich plasma: a comparison of four different systems. Vox Sang 2006;91:135-9. 